We try to publish authors' responses in the same edition with readers' comments. Time constraints might prevent this in some cases. The problem is compounded in a bimonthly journal where continuity of comment and redress are difficult to achieve. When the redress appears 2 months after the comment, 4 months will have passed since the article was published. Therefore, we would suggest to our readers that their correspondence about published papers be submitted as soon as possible after the article appears.

\section{Severity-Based Classification of Mood and Anxiety Symptoms}

To the Editor: I wish to respond to the obviously wellintentioned but eccentric article by Nease et al, ${ }^{1}$ as well as to the provocative accompanying piece by Schwenk. ${ }^{2}$ It would be difficult not to agree in principle that the existing mental health industry has indeed committed the cardinal error of misclassification: failure to categorize entities according to essential attributes. I noted with disappointment, however, that Nease et al have fared little better, attempting, for instance, to explore co-occurrence of mood or anxiety symptoms without any serious mention of such crucially contributory, if not partially causal, issues as street drug abuse, family violence, and the like.

The observations concerning sociodemographics, by which the authors presumably mean to imply downward socioeconomic mobility, are couched in statistical jargon and disparate mathematical methods, which, quite frankly, I suspect are beyond the technical comprehension of most of your readership, myself included. I see no concession that, however statistically valid the classification model of Nease et al might be, every seasoned family physician knows that downward sociodemographic change by any other name still tends for the most part to be a one-way street that is not easily remedied, if at all, by the comforting fiction of generous doses of SSRIs and the well-meaning administration of depression self-adjustment scales, stress management patient handouts, and the like. In any ordinary family practice waiting room, the seasoned observer can overhear the real conceptual common denominators of behavioral medicine: worry (not generalized anxiety) about family substance abuse; fear and intimidation by verbal or often worse domestic violence; and sadness (not media-generated word inflation, ie, depression) about bad, volitional choices, such as money spent on lottery tickets, sexual misadventures, and broken commitments.

My hopes were temporarily lifted by the perceptive edge to Schwenk's editorial, but when my eyes found the words "we are prisoners of how medical problems are named," I was, as so many times in the trendy recent past, confronted by yet another postmodern bromide, promulgated without evidence as if it had been a long-neglected axiom that should really have been served for centuries as an adjunct to mother's milk. But this is precisely the problem, deftly if innocently inverted, toward which Nease et al gesture. Failure to categorize adequately does not entail a prisoner-of-language conundrum from which there is no escape. As physicians we have the resources and the responsibility to go back to nature and take a fresh look at the data when our classification schemes (well-known to have been death-by-politically-correct committee in case of DSM-IV) collide with reality.

The philosophically and practically self-defeating dogma that any classification implying a better casting of "anxiety" and "depression" still is doomed to a linguistic dead end or cannot avoid the risk of labeling a patient unfairly is deterministic. Simple logic, applied to the inescapable and happy fact that some patients get better, especially those who take responsibility for their lives (as opposed to invoking a face-saving, diagnostic self-explanation for their unfortunate sociodemographics), leads us to conclude that neither physicians nor patients need be so self-fulfillingly pessimistic. The perhaps unintended implications of both writings were, alas, precisely the fatalistic demoralization that a patient, struggling already for a sense of autonomy, does not need.

Walter Wahl, MD

Bassett - St. Elizabeth Family Medicine Residency Program Utica, NY

\section{References}

1. Nease DE Jr, Volk RJ, Cass AR. Investigation of a severitybased classification of mood and anxiety symptoms in primary care patients. J Am Board Fam Pract 1999;12:21-31.

2. Schwenk TL. The tyranny of names in mental health care. J Am Board Fam Pract 1999;12:99-101.

The above letter was referred to the authors of the articles in question, who offer the following reply.

To the Editor: I appreciate the opportunity to respond to Dr. Wahl's comments on our article "Investigation of a Severity-based Classification of Mood and Anxiety Symptoms. in Primary Care Patients." Dr. Wahl expresses disappointment that our article appears to substitute one imperfect classification scheme for another. I agree wholeheartedly that classification schemes are at best blunt instruments and no substitute for seasoned family physicians' experience and judgment. Once one moves beyond the office for purposes of communication with colleagues, third party payers, and those in research and health policy, however, they are unavoidable.

Herein lies the rub. If the classification scheme suffers from having been created in a different clinical context where the recognition issues are different, the blunt in- 
strument can be wielded with unfortunate results when it is applied to our discipline. Family physicians then become like the confused individual who is told that a zebra must be a tiger because it has vertical stripes, never mind the other differences! I would argue that if a better classification scheme can be developed that is more true to the patients sitting in the "ordinary family practice waiting room," we would be foolhardy to eschew its use.

Donald E. Nease, Jr, MD University of Michigan Health System Ann Arbor

To the Editor: The point Dr. Wahl seems to be making is that experienced family physicians should feel free to direct that experience toward the accurate, naturalistic description of mental illness phenomena in a way which is beneficial for the patient so afflicted. The words and labels used are not only irrelevant, but they can be harmful if they lead to inappropriate treatment or a pessimistic prognosis that is unwarranted. I could not agree more.

Thomas L. Schwenk, MD

University of Michigan Health System Ann Arbor

\section{Screening and Counseling for Down Syndrome}

To the Editor: I found Drs. Cate and Ball's review of screening for Down syndrome (Cate S, Ball S. Multiple marker screening for Down syndrome - Whom should we screen. J Am Board Fam Pract 1999;12:367-74) informative and sensitive. I believe, however, its advice that "pretest counseling should be nondirective" oversimplifies complex and difficult issues.

I have a 16-year-old daughter, Kate, with Down syndrome and have thought long about prenatal diagnosis. Being told that my first-born child would be disabled was terribly painful. Raising her has been a difficult challenge. I know personally the emotional, physical, and financial costs in raising a disabled child. But, as the review suggests, there have also been great gifts. She is a delightful young woman, and she is delighted with herself. In loving her and being loved by her, I have learned that being accomplished is not what makes people worthwhile, that intelligence is not the measure of a person's soul or a person's joy or a person's humanity. In accepting her fully, I have slowly come to accept myself more fully, with all my human flaws and struggles. I lose track of this still, sometimes daily, in our culture that so much judges and values people by their beauty, wealth, and ability. Kate is there every day reminding me, making me smile, a living contradiction to my confusions.

Our technology now allows us to screen fetuses and offer patients the choice of eliminating some who are impaired. If I had been offered this choice with Kate, I don't know what I would have done. I fear I would have been overwhelmed and turned away from having her. That path on its face seems easier, but has a high price the price of knowing every day that I chose to do away with a child of mine rather than accept her. It leaves me less forgiving and accepting of my children, the world, and myself.
I think the counseling of patients regarding prenatal diagnosis (with termination) requires that we direct patients to explore their values about disability and human life. This task is daunting (particularly in a 15-minute visit!). I believe my patients need help at such times to work through their feelings about this very painful prospect and discover their heart's choice. In this counseling I hope to be nonjudgmental and listen well. I also want to challenge assumptions and help them look deeply.

David E. Nicklin, MD

Univeristy of Pennsylvania

Philadelphia

The above letter was referred to the authors of the article in question, who offer the following reply.

To the Editor: We appreciate Dr. Nicklin's personal reflections. We hope our article continues to stimulate discussion about the appropriate application of such screening tests as the multiple marker screening test. In this article we hoped to convey to the busy physician what we believed were the minimum requirements for adequate pretest counseling. It is clear that nondirective counseling is fundamental if a patient is to reach a decision regarding screening that is most consistent with her values and desires. It is also clear from reading the literature that physicians do not do a very good job of this type of counseling. ${ }^{1}$ Our guideline for pretest counseling is intentionally simplified in an effort to assist physicians in trying to fit this discussion into a 15 -minute visit. We agree it is difficult.

Sara Cate, MD
Yakima Valley Memorial Hospital
Susie Ball, MD
Central Washington Family Medicine
Residency Program
Yakima, Wash

\section{References}

1. Braddock $\mathrm{CH} 3 \mathrm{~d}$, Edwards KA, Hasenberg NM, Laidley TL, Levinson W. Informed decision making in outpatient practice: time to get back to basics. JAMA 1999;282:2313 20.

\section{Family Physicians and Firearm Safety Counseling}

To the Editor: Shaughnessy and colleagues, ${ }^{1}$ in their article on family physicians and firearm safety counseling, have clarified a long-neglected question: how do patients view physicians' counseling on firearms?

Patients have good reasons to view such counseling with skepticism. The American Medical Association (AMA), ${ }^{2}$ the American Academy of Pediatrics (AAP), ${ }^{3}$ and the American College of Physicians (ACP) ${ }^{4}$ have all adopted and publicized policies urging more gun control. These policies range from even higher tax burdens on gun owners to support for handgun bans. All these organizations advocate legislation hostile to gun owners.

None of these organizations or physicians firearm experts attempt to reconcile or even acknowledge the existing body of criminology research on firearms. Most 